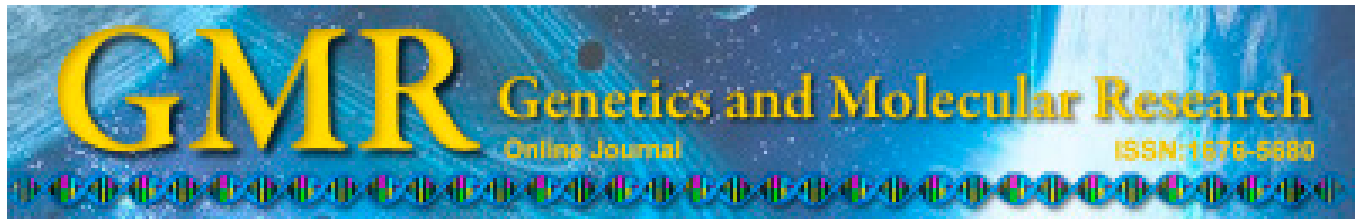

\title{
Expression of COX-2 and VEGF-C in cholangiocarcinomas at different clinical and pathological stages
}

\author{
Z. You, L. Bei, L.P. Cheng and N.S. Cheng \\ Biliary Surgery, West China Hospital, Chengdu, China \\ Corresponding author: N.S. Cheng \\ E-mail: yurihobadehoya@126.com
}

Genet. Mol. Res. 14 (2): 6239-6246 (2015)

Received August 20, 2014

Accepted January 19, 2015

Published June 9, 2015

DOI http://dx.doi.org/10.4238/2015.June.9.9

\begin{abstract}
The aim of this study was to investigate the expression and clinical significance of cyclooxygenase 2 (COX-2) and vascular endothelial growth factor C (VEGF-C) in cholangiocarcinomas at different clinical and pathological stages. Eighty cholangiocarcinoma samples of patients treated with surgery between January 2012 and January 2014 were collected. Immunohistochemistry was used to detect COX-2 and VEGF-C expression at different clinical and pathological stages. ELISA, real-time PCR, invasive chambers, and MTT assay were applied in cultured cholangiocarcinoma cells treated with a COX-2 inhibitor. Expression of COX-2 and VEGF-C correlated positively with the clinical TNM stage but did not correlate with the differentiation status. Inhibition of COX-2 activity reduced VEGF-C mRNA expression and secretion in cholangiocarcinoma cells and decreased their migration but not proliferation. Because of its ability to inhibit invasion, COX-2 could be a new target for treatment of cholangiocarcinoma.
\end{abstract}

Key words: Cholangiocarcinoma; Clinical staging;

Cyclooxygenase 2; Vascular endothelial growth factor $\mathrm{C}$ 


\section{INTRODUCTION}

Cholangiocarcinoma is a common malignant gastrointestinal tumor whose clinical characteristics include slowly progressing symptoms, invasiveness, low radical resection rate, and insensitivity to chemoradiotherapy. Its prognosis is generally poorer than other malignancies such as gastric cancer and colorectal cancer (Fava and Lorenzini, 2012; Aishima et al., 2013; Marino et al., 2013). Thus, it is important to identify new treatments for cholangiocarcinoma. Owing to in-depth research on tumorigenesis and progression, the angiogenic characteristics of cholangiocarcinoma have gained attention. Indicators of tumor angiogenesis such as cyclooxygenase-2 (COX-2) and vascular endothelial growth factor C (VEGF-C) are overexpressed in many types of malignant tumors (Zabron et al., 2013). However, the role of COX-2 and VEGF-C in cholangiocarcinoma is still unclear, especially in respect to clinical stage. The present study assesses COX-2 and VEGF-C expression at different cholangiocarcinoma stages and the clinical significance and interrelationship of these proteins in this disease.

\section{MATERIAL AND METHODS}

\section{Clinical samples}

Eighty cholangiocarcinoma specimens of patients treated with surgery between January 2012 and January 2014 were analyzed. None of the patients had received preoperative chemoradiotherapy or immune therapy. The clinical stage of the specimen was based on the TNM staging criteria for cholangiocarcinoma issued by the American Cancer Society (Zabron et al., 2013). Twenty-six cases were stage I, 30 were stage II, 19 were stage III, and 5 were stage IV. According to standard pathological tissue differentiation criteria, 37, 25, and 18 tumors were well, moderately, and poorly differentiated, respectively. Twenty-five specimens from non-tumor tissue (gall bladder tissue from patients with gallstones or cholecystitis) served as the controls.

\section{Immunohistochemistry}

Sections from paraffin blocks were cut and mounted onto adhesive-coated glass slides. Slides were incubated successively in xylene (thrice for 5 min each), absolute ethanol (twice for 3 min each), 95\% ethanol (twice for 3 min each), and distilled water (a minimum of 30 s). Specific antibodies for COX-2 and VEGF-C immunostaining were performed. Gastric cancer tissue samples were used as the positive control, and samples incubated with phosphate-buffered saline instead of primary antibody served as the negative control. Positive immunostaining was indicated by the presence of brown particles in the cytoplasm. The semi-quantitative evaluation method was as follows: 3 points for $>60 \%$ positive cells, 2 points for 30 to $59 \%$ positive cells, 1 point for 10 to $29 \%$ positive cells, and 0 points for $<10 \%$ positive cells.

To determine tumor microvascular density (MVD), microvascular endothelial cells were analyzed for CD34 by using immunohistochemistry. Brown or claybank cells were considered positive. The four areas with the highest blood vessel density were selected, and positive endothelial cell clusters were counted. Each cell cluster was considered a microvessel, and MVD was the mean number of microvessels in the four areas. 


\section{Cell culture}

The human cholangiocarcinoma cell line QBC939 was obtained from Boster Biotechnology (Wuhan, China) and maintained in RPMI-1640 medium (Life Technologies), supplemented with $10 \%$ fetal calf serum (Gibco, Grand Island, NY, USA) in a humidified atmosphere containing $5 \% \mathrm{CO}_{2}$ at $37^{\circ} \mathrm{C}$.

\section{Cancer cell performance tests}

\section{Cell proliferation assay}

Cells in logarithmic phase were seeded on 96-well plates at a density of $1 \times 10^{4}$ cells/ well. Twenty-four hours after seeding, cells were incubated in the medium containing the COX-2 inhibitor NS-398 at concentrations ranging from 0 to $200 \mu \mathrm{M}$ for 24,48 , or $72 \mathrm{~h}$ at $37^{\circ} \mathrm{C}$. 3-(4,5-Dimethylthiazol-2-yl)-2,5-diphenyltetrazolium bromide (MTT) (5 mg/mL, 20 $\mu \mathrm{L}$ ) was added, and the cells were incubated for $4 \mathrm{~h}$ at $37^{\circ} \mathrm{C}$ before addition of dimethylsulfoxide. Absorbance at $490 \mathrm{~nm}$ (A490) was read for each well using a spectrophotometer. The inhibition rate was calculated as 1 - A490 (test group)/A490 (control). Three independent experiments were performed with five wells per data point.

\section{Cell invasion assay}

Cell invasion was assayed using Matrigel Coated Invasion Chambers (BD, CA, USA). Cells $\left(2 \times 10^{5} /\right.$ well $)$ were added to the inside of the inserts and incubated with or without NS398 for $36 \mathrm{~h}$. After incubation, non-invading cells were removed from the upper surface of the membrane using cotton-tipped swabs. The cells on the lower surface of the membrane were stained with crystal violet and counted in the central fields of triplicate membranes.

\section{VEGF-C secretion and $m R N A$ expression assays}

Cells in logarithmic phase were seeded on 24-well plates and incubated with 0 to 200 $\mu \mathrm{M}$ NS-398 for $24 \mathrm{~h}$ at $37^{\circ} \mathrm{C}$. The medium was collected and centrifuged for $10 \mathrm{~min}$ at 10,000 rpm at $4^{\circ} \mathrm{C}$. VEGF-C in the supernatant was detected by using enzyme-linked immunosorbent assay, according to the instructions in the operation manual (USCN Life Science Inc, Wuhan, China). RNA was extracted from cells using Trizol (Takara). Real-time polymerase chain reaction (PCR) was used to quantitate VEGF-C mRNA expression using the following primers: 5'-CGCTGCTGCCTGCGCTCTG-3' (forward) and 5'-AGCCACCTCCTGGATGGTC-3' (reverse).

\section{Statistical analysis}

Numerical data are reported as means \pm standard error. Differences between groups were analyzed by using the Student $t$-test or ANOVA. All statistical analyses were performed using the SPSS 17.0 software (Chicago, IL, USA). P values $<0.05$ were considered to be statistically significant. 


\section{RESULTS}

\section{COX-2 and VEGF-C expression in cholangiocarcinoma tissue}

COX-2 and VEGF-C expression in 80 cholangiocarcinoma specimens were semiquantified by using immunohistochemistry. For both proteins, the percentage of stained cells increased significantly with increasing clinical stage $(\mathrm{P}<0.05)$ (Table 1). On the other hand, the percentage of stained cells increased slightly, but this change was not significant $(\mathrm{P}>0.05)$. These findings suggest that expression of COX-2 and VEGF-C correlates with clinical stage but not pathological stage.

Table 1. Correlation between cyclooxygenase 2 (COX-2) and vascular endothelial growth factor C (VEGF-C) expression and cholangiocarcinoma clinical and pathological stages (number \pm standard error).

\begin{tabular}{llll}
\hline Item & COX-2 & VEGF-C & MVD \\
\hline Clinical stage (TNM) & & & \\
$\quad$ Stage I & $1.21 \pm 0.35$ & $1.52 \pm 0.28$ & $32.14 \pm 7.18$ \\
Stage II & $1.29 \pm 0.37$ & $1.59 \pm 0.26$ & $42.33 \pm 8.16$ \\
Stage III & $2.32 \pm 0.42$ & $2.52 \pm 0.32$ & $59.52 \pm 11.35^{*}$ \\
Stage IV & $2.78 \pm 0.21^{*}$ & $2.92 \pm 0.06^{*}$ & $55.35 \pm 10.28^{*}$ \\
Pathological stage & & & \\
$\quad$ Well & $1.99 \pm 0.26$ & $1.96 \pm 0.52$ & $44.26 \pm 9.9$ \\
$\quad$ Moderate & $2.01 \pm 0.42$ & $2.12 \pm 0.41$ & $46.54 \pm 10.05$ \\
Poor & $2.21 \pm 0.17$ & $2.24 \pm 0.05$ & $47.79 \pm 8.47$ \\
\hline
\end{tabular}

$* \mathrm{P}<0.05$ compared with values in TNM stage I and II.

\section{Microvascular formation in cholangiocarcinoma tissue}

MVD in cholangiocarcinoma tissue was significantly higher at stage III and IV than stage I and II (Table 1). Moreover, it also correlated positively with VEGF-C expression ( $\mathrm{r}=0.776, \mathrm{P}<$ 0.01). COX-2 expression was also correlated positively with MVD $(r=0.623, \mathrm{P}<0.05)$.

\section{Effect of COX-2 inhibition on VEGF-C expression in QBC939 cells}

QBC939 cholangiocarcinoma cells were treated with various concentrations of the COX-2 inhibitor NS-398, and amounts of VEGF-C mRNA and secreted VEGF-C were determined. Both VEGF-C mRNA expression (Figure 1) and secretion (Figure 2) were lower in inhibitor-treated cells than untreated cells. These results suggest that COX-2 can regulate VEGF-C secretion.

\section{Effect of COX-2 inhibition on cell proliferation and invasion}

MTT incorporation was observed in QBC939 cells treated with or without various concentrations of with NS-398 for 24, 48, or $72 \mathrm{~h}$; the differences were not significant (Table 2). This finding suggests that COX-2 does not significantly influence cell proliferation. On the other hand, the number of cells invading the matrigel in cell invasion chambers decreased significantly in the presence of NS-398 concentrations above $100 \mu \mathrm{M}$ (Table 3). This finding indicates that cell invasion requires COX-2 activity. 


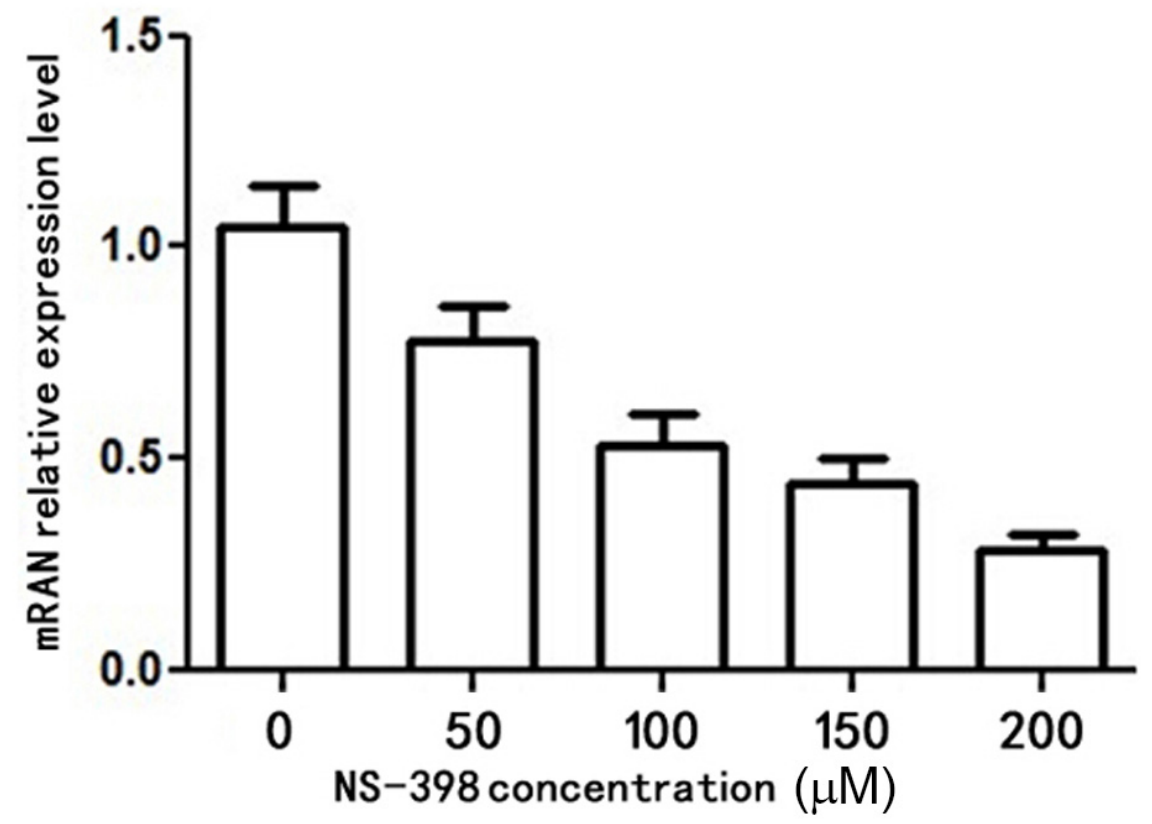

Figure 1. Vascular endothelial growth factor C (VEGF-C) mRNA expression was determined by using real-time polymerase chain reaction. As NS-398 concentrations increased, VEGF-C mRNA levels decreased.

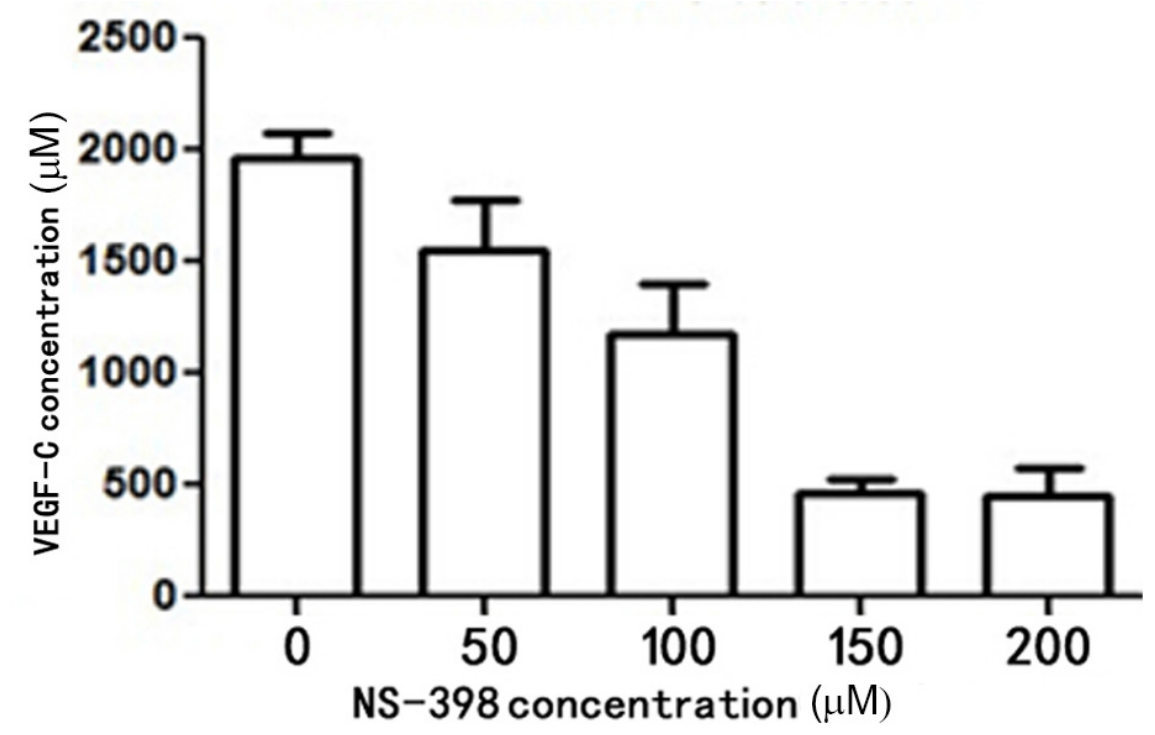

Figure 2. Vascular endothelial growth factor C (VEGF-C) secretion was determined by using enzyme-linked immunosorbent assay. As NS-398 concentrations increased, levels of VEGF-C in the culture medium decreased. 
Table 2. Effect of cyclooxygenase inhibition on QBC939 proliferation (percentage \pm standard error).

\begin{tabular}{llll}
\hline NS-398 $(\mu \mathrm{M})$ & $24 \mathrm{~h}$ IR $(\%)$ & $48 \mathrm{~h} \mathrm{IR}(\%)$ & $72 \mathrm{~h}$ IR $(\%)$ \\
\hline 50 & $19.39 \pm 4.29$ & $21.33 \pm 5.18$ & $21.98 \pm 4.28$ \\
100 & $20.41 \pm 4.28$ & $23.54 \pm 6.02$ & $20.16 \pm 3.29$ \\
150 & $22.15 \pm 5.02$ & $22.92 \pm 5.35$ & $22.03 \pm 4.35$ \\
200 & $21.97 \pm 4.82$ & $21.03 \pm 4.78$ & $23.51 \pm 4.26$ \\
\hline
\end{tabular}

IR: inhibition rate.

Table 3. Effect of cyclooxygenase inhibition on QBC939 invasion (number \pm standard error).

\begin{tabular}{lc}
\hline NS-398 $(\mu \mathrm{M})$ & Cell number at $36 \mathrm{~h}$ \\
\hline 50 & $32.58 \pm 3.69$ \\
100 & $26.44 \pm 3.07$ \\
150 & $17.14 \pm 2.52$ \\
200 & $11.03 \pm 2.76$ \\
\hline
\end{tabular}

\section{DISCUSSION}

COX-2 is an important enzyme that converts arachidonic acid to prostaglandins. In recent years, many studies have shown that COX-2 is overexpressed in bladder, non-small cell lung, and gastric cancers (Grammas, 2011; Fukuhara et al., 2013; Rosas et al., 2014; Thomas, 2014). Additional studies showed that COX-2 expression was closely associated with local angiogenesis, tumor cell infiltration, proliferation, and apoptosis (Grammas, 2011; Fava and Lorenzini, 2012; Fukuhara et al., 2013; Rosas et al., 2014). VEGF is the most important factor in angiogenesis and has been widely investigated. Our study focused on cholangiocarcinoma, a cancer in which COX-2 and VEGF-C expression have been less studied, particularly in terms of clinical stage and interrelationship.

Although not normally expressed, COX-2 is upregulated in response to inflammation and in tumors (Grammas, 2011). Previous studies of COX-2 expression in gallbladder were mostly limited to comparisons between the gallbladder and other tissues (Fukuhara et al., 2013; Rosas et al., 2014). Consistent with previous studies, COX-2 expression was significantly higher in tumor tissue than benign lesions (gallbladder tissue from patients with cholecystitis and gallstone) in our study. Considering the high invasive characteristics of tumorigenesis and clinical treatments, we compared COX-2 expression at different clinical and pathological stages. Clinical stages are defined on the basis of tumor infiltration, while pathological stages are defined on the basis of cell differentiation. Moreover, the former directly reflects tumor invasion status, while the latter indicates degree of malignancy. Our immunohistochemistry results showed that COX-2 expression in cholangiocarcinoma tissue increased along with both the clinical stage and pathological stage. This result indicates that COX-2 is closely associated with poorly differentiated, highly invasive cholangiocarcinomas.

Tumor angiogenesis-targeted therapy has become popular in recent years and has achieved significant curative effects in non-small cell lung cancer. Inhibitors such as the VEGF monoclonal antibody showed significant clinical effects (Ding et al., 2012). In the present study, MVD in cholangiocarcinoma tissue was significantly higher at later than earlier clinical and pathological stages. Thus, angiogenesis occurs during cholangiocarcinoma progression. Similar to COX-2 expression and MVD, VEGF expression was also higher at later clinical stages, as determined by using immunohistochemistry and real-time PCR. These results sug- 
gest that VEGF-C expression and secretion gradually increase as cholangiocarcinomas progress. Because VEGF-C can induce vascular endothelial cells formation and expansion in solid tumor and surrounding tissue, and improve permeability, cancer cells overexpressing VEGF-C have a higher chance of crossing microvessels and metastasizing through the blood. At the same time, VEGF-C can promote local matrix reconstruction and endothelial cell displacement, leading to the formation of new blood vessels. Therefore, high expression of VEGF-C is necessary for the growth of tumors with rapid metabolism and high oxygen demand. Our data showing that VEGF-C expression increases along with clinical and pathological stages confirm previous results (Schoppmann et al., 2011; Sirica, 2011; Bai et al., 2014).

Compared with other cancers, cholangiocarcinoma responds poorly to chemotherapy. Therefore, it has important to discover new drugs for treating cholangiocarcinoma and to evaluate their mechanisms (Malaguarnera et al., 2011). In recent years, nonsteroidal anti-inflammatory drugs such COX-2-selective inhibitors have been extensively used in clinical treatments. COX-2 inhibitors have been reported to effectively inhibit the growth of colon, breast, lung, and prostate tumors (Wu et al., 2010; Fukuhara et al., 2013). However, in the present study, the COX-2 inhibitor NS-398 did not significantly inhibit the proliferation of cholangiocarcinoma cells in vitro. This finding is similar to those in previous reports of COX-2 expression and function at different stages of cholangiocarcinoma (Wu et al., 2010; Yoon et al., 2010; Rodriguez-Enriquez et al., 2011; Schoppmann et al., 2011). On the other hand, COX-2 inhibitors have been confirmed as effective auxiliary drugs for cholangiocarcinoma (Murmu, 2014; Zabron et al., 2013). Thus, we focused on cell invasion and found that inhibition of COX-2 activity reduced the invasion ability of cultured cholangiocarcinoma cells. This suggests that COX-2 is mainly associated with invasion in cholangiocarcinoma and may explain why COX-2 is more highly expressed at later clinical and pathological stages.

VEGF-C is an important treatment target for numerous tumors, and COX-2 is an important regulatory factor of VEGF-C expression (Wiedmann and Mossner, 2010). Upregulation of COX-2 in colon cancer can enhance VEGF-C expression, whereas downregulation of COX-2 has the opposite result (Granci et al., 2010). To investigate the relationship between COX-2 and VEGF-C in cholangiocarcinoma, we measured VEGF-C mRNA levels and secretion in cholangiocarcinoma cells treated with a COX-2 inhibitor. Inhibition of COX-2 activity reduced VEGF-C mRNA levels and levels of secreted VEGF-C. Because VEGF-C is a cytokine that promotes local microvascular formation and tumor cell invasion, our results suggest that COX-2 increases these processes via upregulation and consequent secretion of VEGF-C.

\section{CONCLUSIONS}

The present study analyzed COX-2 and VEGF-C expression in cholangiocarcinoma using clinical specimens and a cell line. Our results showed that COX-2 and VEGF-C expression in cholangiocarcinoma tissue positively correlated with clinical TNM stage but not cancer cell differentiation. Inhibition of COX-2 activity reduced amounts of VEGF-C RNA and secreted VEGF-C, indicating that VEGF-C expression requires COX-2 activity, and decreased migration but not proliferation in cholangiocarcinoma cells. COX-2 can be used as a new treatment target for cholangiocarcinoma owing to its ability to inhibit tumor cell metastasis. 


\section{ACKNOWLEDGMENTS}

Research supported by the Science Foundation of Sichuan Province (\#2014JY0223).

\section{REFERENCES}

Aishima S, Mano Y, Tanaka Y, Kubo Y, et al. (2013). Different roles of inducible nitric oxide synthase and cyclooxygenase-2 in carcinogenesis and metastasis of intrahepatic cholangiocarcinoma. Hum. Pathol. 44: 1031-1037.

Bai X, Yang Q, Shu W, Wang J, et al. (2014). Prostaglandin E2 upregulates beta-1 integrin expression via the E prostanoid 1 receptor/nuclear factor beta-light-chain-enhancer of activated B cells pathway in non-small-cell lung cancer cells. Mol. Med. Rep. 9: 1729-1736.

Ding M, Fu X, Tan H, Wang R, et al. (2012). The effect of vascular endothelial growth factor C expression in tumorassociated macrophages on lymphangiogenesis and lymphatic metastasis in breast cancer. Mol. Med. Rep. 6: 10231029.

Fava G and Lorenzini I (2012). Molecular pathogenesis of cholangiocarcinoma. Int. J. Hepatol. 2012: article ID 630543, 7 pages.

Fukuhara J, Kase S, Ohashi T, Ando R et al. (2013). Expression of vascular endothelial growth factor C in human pterygium. Histochem. Cell Biol. 139: 381-389.

Grammas P (2011). Neurovascular dysfunction, inflammation and endothelial activation: implications for the pathogenesis of Alzheimer's disease. J. Neuroinflammation 8: 26.

Granci V, Dupertuis YM and Pichard C (2010). Angiogenesis as a potential target of pharmaconutrients in cancer therapy. Curr. Opin. Clin. Nutr. Metab. Care 13: 417-422.

Malaguarnera G, Giordano M, Paladina I, Rando A, et al. (2011). Markers of bile duct tumors. World J. Gastrointest. Oncol. 3: 49-59.

Marino D, Leone F, Cavalloni G, Cagnazzo C, et al. (2013). Biliary tract carcinomas: from chemotherapy to targeted therapy. Crit. Rev. Oncol. Hematol. 85: 136-148.

Murmu N (2014). Wnt/ $\beta$-Catenin and its downstream signalling as a biomarker at transcriptional and post transcriptional level in liver cancer. Mol. Cancer Biol. 2, in press.

Rodriguez-Enriquez S, Pacheco-Velazquez SC, Gallardo-Perez JC, Marín-Hernández A, et al. (2011). Multi-biomarker pattern for tumor identification and prognosis. J. Cell. Biochem. 112: 2703-2715.

Rosas C, Sinning M, Ferreira A, Fuenzalida M, et al. (2014). Celecoxib decreases growth and angiogenesis and promotes apoptosis in a tumor cell line resistant to chemotherapy. Biol. Res. 47: 27.

Schoppmann A, Tamandl D, Herberger B, Längle F, et al. (2011). Comparison of lymphangiogenesis between primary colorectal cancer and corresponding liver metastases. Anticancer Res. 31: 4605-4611.

Sirica AE (2011). The role of cancer-associated myofibroblasts in intrahepatic cholangiocarcinoma. Nat. Rev. Gastroenterol. Hepatol. 9: 44-54.

Thomas MB (2014). Systemic and targeted therapy for biliary tract tumors and primary liver tumors. Surg. Oncol. Clin. N. Am. 23: 369-381.

Wiedmann MW and Mossner J (2010). Molecular targeted therapy of biliary tract cancer-results of the first clinical studies. Curr. Drug Targets. 11: 834-850.

Wu WK, Sung JJ, Lee CW, Yu J, et al. (2010). Cyclooxygenase-2 in tumorigenesis of gastrointestinal cancers: an update on the molecular mechanisms. Cancer Lett. 295: 7-16.

Yoon BI, KimYH, Yi JY, Kang MS, et al. (2010). Expression of thioredoxin during progression of hamster and human cholangiocarcinoma. Cancer Sci. 101: 281-288.

Zabron A, Edwards RJ and Khan SA (2013). The challenge of cholangiocarcinoma: dissecting the molecular mechanisms of an insidious cancer. Dis Model Mech. 6: 281-292. 